\title{
Experimental investigation of unbound nodes identification for metallic sandwich panels with truss core
}

\author{
Lingling Lu, Hongwei Song*, Chenguang Huang \\ Key Laboratory for Mechanics in Fluid Solid Coupling Systems, Institute of Mechanics, Chinese Academy of Science, Beijing 100190, China \\ School of Engineering Science, University of Chinese Academy of Sciences, Beijing 100049, China
}

\section{A R T I C L E I N F O}

\section{Article history:}

Received 11 June 2016

Revised 1 November 2016

Accepted 7 December 2016

Available online 9 December 2016

\section{Keywords:}

Sandwich panel with truss core

Damage identification

Unbound nodes

Teager energy operator

\begin{abstract}
A B S T R A C T
Experimental investigation of metallic pyramidal sandwich panels with truss core in the cases of different extent and location of unbound nodes damages are conducted and a baseline-free damage identification method based on flexible matrix, gapped smoothing method and Teager energy operator is used. The influences of sensor density and Teager energy operator on the damage identification are also discussed. Moreover, a $0-1$ unbound nodes identification method is also proposed to capture the shape and size of the unbound nodes damage zone. The results show that the proposed baseline-free method could identify the unbound nodes damage of metallic sandwich panels with truss core effectively. Increasing sensor density is beneficial for damage identification, and there is a critical sensor density for the identification of unbound nodes damage. Teager energy operator plays a very important role in suppressing fluctuations and singularities caused by non-damage factors, such as boundary condition, noise and shaker. The identified damage zone obtained by the $0-1$ method is in accordance with the actual damage zone.
\end{abstract} () 2016 Elsevier Ltd. All rights reserved.

\section{Introduction}

As a class of newly developed structures, sandwich structures consisting of two thin face sheets and a lightweight core [1-3] have many superior properties, including high specific bending stiffness, light weight, good thermal insulation and acoustical isolation [47], and are considered as very promising candidates to be applied in the thermal protection systems (TPS) of high-speed aircrafts. Among them, sandwich panels with truss core (SPTCs) are recently proposed and fabricated $[3,8]$. However, due to the immaturity of fabrication technology and high temperature service environment $[9,10]$, defects and damages may occur in SPTCs, such as unbound truss nodes to the face sheet, buckling of the panels [11,12], burnthough of face sheets and breakage of truss member and so on. The defects or damages may alter mechanical properties of SPTCs, such as dynamic properties (natural frequencies and modes) [13], the stiffness and strength [14], and the load capacity [15]. Unbound

\footnotetext{
* Corresponding author at: Key Laboratory for Mechanics in Fluid Solid Coupling Systems, Institute of Mechanics, Chinese Academy of Science, Beijing 100190, China; School of Engineering Science, University of Chinese Academy of Sciences, Beijing 100049, China.

E-mail address: songhw@imech.ac.cn (H. Song).
}

node is the most commonly found defect during fabrication of SPTCs, especially when the total number of nodes is large. For the truss core fabrication method like punching of the perforated metal sheet, it is difficult to guarantee every node perfectly bonded to the face sheets.

Russell and Hilary [16] proposed that it is necessary to quantify the SPTCs' sensitivity to imperfections before they can be implemented as structural components in the field. They investigated the retention of stiffness and load capacity in the presence of unbound nodes. Results show that pyramidal truss core sandwich structures are robust under compressive load. But the imperfections would cause rapid degradation of core shear properties. Evans et al. [8] and Wadley et al. [3] also studied the effect of defects on the mechanical properties of cellular metal sandwich structures. Wallach and Gibson [14] investigated the influence of randomly removing members of a three-dimensional truss material on the Young's modulus and compressive strength.

Meanwhile, works on the effect of damages and defects on the dynamic properties of sandwich structures are also carried out. The effect of delamination on free vibration of composite sandwich beams is investigated theoretically by $\mathrm{Hu}$ and Hwu [17]. Kim and Hwang [18] studied the influence of debonding extent on flexural stiffness, natural frequencies and frequency response functions of composite honeycomb sandwich beam. They found 
that the flexural stiffness and natural frequency would decrease as the debonding emerges or the extent of debonding increases. Sokolinsky et al. [19] analysed the free vibration of sandwich beam with a locally damaged core by the higher-order theory approach, and results showed that a small local damage would cause significant changes in the natural frequencies and corresponding vibration modes. Burlayenko and Sadowski [20] studied the effect of skin/core debonding on free vibration behavior of foam and honeycomb cored sandwich plates, and the influences of debonding size, location and type on the modal parameters with different boundary conditions were also investigated. Buket and Srinivasa [21] discussed the influence of curvature and face/core debond on the vibration behavior of curved composite sandwich beams. Results indicated that face/core debonding would cause the reduction of the natural frequencies. Lou et al. [22] also investigated the effect of damage extent, damage location, damage form and boundary conditions on the vibration characteristics of composite pyramidal truss core sandwich structures by numerical simulation.

Based on the variations of mechanical properties of sandwich panels caused by damages and defects, some damage identification methods have been developed for sandwich structures. For fiberreinforced polymer (FRP) honeycomb sandwich beams, Lestari and Qiao [23] used the changes of the curvature mode shapes before and after damage to identify the structural damages. $\mathrm{Hu}$ et al. [24] used strain energy calculated by the differential quadrature method to detect surface cracks of various composite laminates. For composite sandwich beam, Kumar et al. [25] presented that the modal strain energies before and after damage can be used to identify both damage location and damage extent. Zhu et al. [26] proposed a non-destructive evaluation (NDE) method based on frequency response function (FRF) measured at one point, and the damage parameters were inversed by simple genetic algorithm. The experimental results demonstrated the method can inverse the damage location and size with acceptable precision. Based on wavelet transform, Andrzej [27] presented a novel approach of two-step damage detection for composite sandwich structures with honeycomb-core. Tian et al. [28] used Uniform Load Surface (ULS) curvature to detect the delamination defects of the lattice sandwich plate. Based on ULS, Li et al. [29] proposed a baselinefree NDE method to determine the debonding of truss bar in composite SPTCs.

In the previous work, much effort has been devoted to explore effective damage identification methods for composite SPTCs. However, little research has been undertaken into damage detection of metallic SPTCs, which is considered as a most promising TPS structure. Due to the immaturity of manufacturing technique, defects may arise and the form of unbound nodes between the core and face sheets is a most typical flaw, which would be enlarged quickly under cyclic loading. Comparing with damages like truss core missing and burn-though of face sheets, unbound nodes are usually invisible and the variation of structural dynamic property caused by this defect is usually much smaller than other damage styles.

In the study, a baseline-free NDE method is used for unbound nodes identification of metallic SPTCs, and experiments of metallic pyramidal SPTCs in the case of different unbound nodes damages are carried out. The fabrication of specimens with unbound nodes is introduced in Section 2. The experimental system and process are illustrated in Section 3. Section 4 introduces the proposed damage identification method. In Section 5, the experimental validation results are presented and the influences of sensor density and Teager energy operator on damage identification are discussed. Besides, a 0-1 unbound nodes identification method is presented to identify the size of unbound nodes zone. In Section 6, some conclusions are obtained.

\section{Specimens preparation}

\subsection{Fabrication of specimens}

Up to now, the fabrication of a sandwich panel with truss core is still in the laboratory-level stage. In the present work, the metallic pyramidal truss cores with a relative density $\rho$ of about $3 \%$ are fabricated from $0.7 \mathrm{~mm}$ perforated stainless steel sheet by folding technique. Fig. 1 illustrates the punching operation to fold the perforated sheet into pyramidal truss cores, by using a punch-and-die pair of $60^{\circ}$ angle. The punch-and-die pair is designed with a $3 \mathrm{~mm}$ terrace to obtain flat areas at nodal regions, in order to avoid node fracture and enlarge the joint area between truss cores and face sheets. The brazing technique, which leaves no flaw on the face sheets, is utilized to join the truss core with face sheets. The dimension of the face sheet is $250 \mathrm{~mm} \times 250 \mathrm{~mm} \times 0.9 \mathrm{~mm}$. The shape of the cross section of truss bar is square and the unit cell of pyramidal truss is shown in Fig. 2, where $h_{c}=7 \mathrm{~mm}, \mathrm{t}_{\mathrm{c}}=0.7 \mathrm{~mm}$, $\mathrm{L}_{1}=15.9 \mathrm{~mm}, \mathrm{~L}_{2}=9.18 \mathrm{~mm}$

\subsection{Specimens with unbound nodes}

In the study, the prefabricated unbound nodes damage is achieved by spreading protective coating on the joint area of the truss core in the predetermined damage zone before brazing, and the diagrammatic sketches is shown in Fig. 3(a), and the example of fabricated damaged specimen is shown in Fig. 3(b). Damages in the case of different locations and sizes are considered. Specimens with damage at the edge are named ED, including ED3 $\times 2$, ED5 $\times 3$ and ED7 $\times 4$, where $x \times y$ represents the damage size, $x$ and $y$ are the numbers of unbound cells in the row and column respectively. Specimens with damage at the structural center are named $C D$, including $C D 3 \times 2, C D 5 \times 3, C D 7 \times 4, C D 9 \times 5$ and $\mathrm{CD} 11 \times 6$. The 8 damaged specimens are as shown in Fig. 4 , and the red rectangle represents the damage zone for unbound nodes.

\section{Experimental setup and modal analysis}

The experimental setup is as shown in Fig. 5. The specimens of metallic SPTC with prefabricated damages are excited by a shaker (JZK-50) and two edges of the specimens are clamped. The excitation signal is generated by the control system of a laser Doppler vibrometer (Polytec, PSV-400) and amplified by a power amplifier (YE5874A) before input to the shaker. The frequency bandwidth of the excitation signal was set in the range of $0-2.5 \mathrm{kHz}$ with the resolution of $1 \mathrm{~Hz}$.

The structural vibration response is measured by the laser Doppler vibrometer. The measuring points are focused on the front sur-

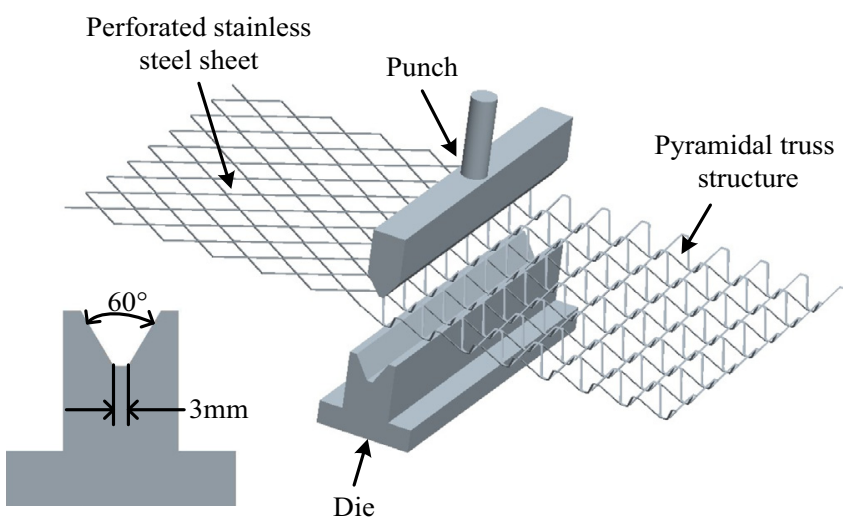

Fig. 1. The punching operation for manufacturing the pyramidal truss cores. 


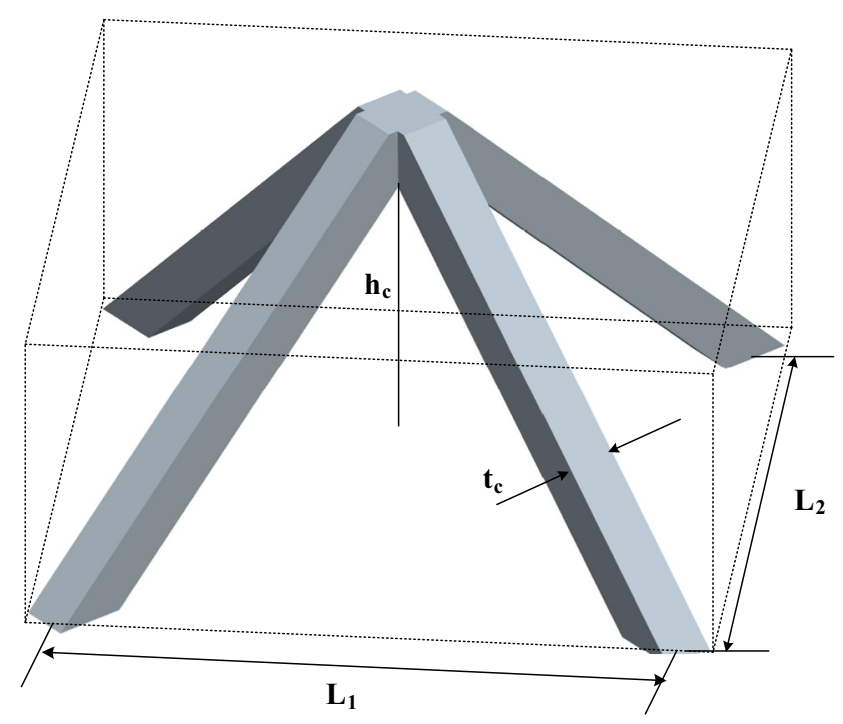

Fig. 2. Unit cell of pyramidal truss.

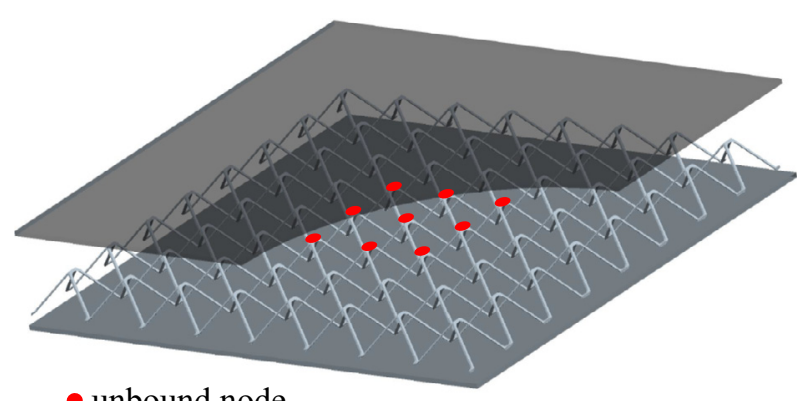

- unbound node

(a)

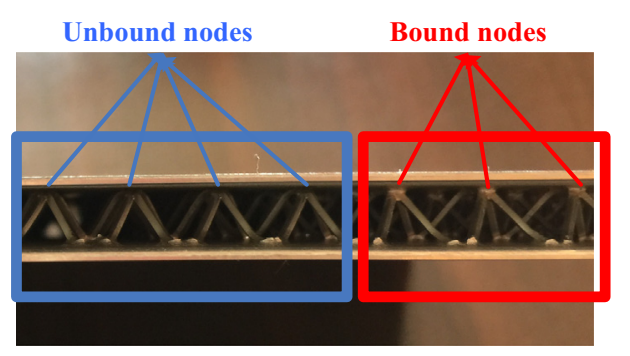

(b)

Fig. 3. Unbound nodes damage. (a) Illustration of protected coating at prefabricated unbound zone. (b) The fabricated damaged specimen.

face of the face sheet, and the excitation is on the other side of the face sheet. Distribution of measuring points of ED5 $\times 3$ specimen is shown in Fig. 6. The frequency response function (FRF) is calculated based on the acquired excitation and structural response signal and the structural modal information could be obtained, including natural frequencies and modes. In order to obtain modal information of good quality, the FRF for each scanning point was averaged three times.

\section{Damage identification method}

In our previous work, a baseline-free damage identification method is proposed for truss core missing of metallic SPTCs [30].
In the present work, the method is improved to identify the unbound nodes damage of metallic SPTCs, as well as the size and location of the unbound nodes. The details are as below.

The contribution of $k$ th order mode for the flexibility matrix can be expressed as

$F_{k}=\frac{\Phi_{k} \Phi_{k}^{T}}{\omega_{k}^{2}}$

where $\omega_{k}$ and $\Phi_{k}$ are the $k$ th order of natural frequency and mass normalized mode respectively.

Then the $k$ th component $M D C_{k}$ of modal deflection of all DOFs under uniform unit load all over the structure can be deduced

$M D C_{k}=\left\{D_{k}(i)\right\}=F_{k} \cdot I$

where $I$ is $\{1, \ldots \ldots, 1\}_{1 * \mathrm{n}}^{\mathrm{T}}$, and $n$ is the number of degrees of freedom (DOFs). It is found that $M D C_{k}$ only relates with the $k$ th order mode and is linear to $\omega_{k}{ }^{-2}$.

Then gapped smoothing method (GSM) is used to identify SPTCs' damages without baseline [31,32]. Eight neighboring points around point $\left(x_{i}, y_{j}\right)$ are selected to fit the $M D C_{k}$ value of point $\left(x_{i}, y_{j}\right)$ (Fig. 7) and the estimated $M D C_{k}\left(x_{i}, y_{j}\right)^{\prime}$ could be expressed as

$$
\begin{aligned}
\operatorname{MDC}_{k}\left(x_{i}, y_{j}\right)^{\prime}= & a_{0}+a_{1} x_{i}+a_{2} y_{j}+a_{3} x_{i} y_{j}+a_{4} x_{i}^{2}+a_{5} y_{j}^{2}+a_{6} x_{i}^{2} y_{j} \\
& +a_{7} x_{i} y_{j}^{2}
\end{aligned}
$$

where $a_{0}$ to $a_{7}$ are the eight fitting coefficients of point $\left(x_{i}, y_{j}\right)$ for the $k$ th component $M D C_{k}$, expressed as $A\left(x_{i}, y_{j}\right)^{k}=\left\{a_{0}, a_{1},,,,, a_{6}, a_{7}\right\}_{1 \times 8}^{\mathrm{T}}$, and they could be deduced as

$A\left(x_{i}, y_{j}\right)^{k}=C\left(x_{i}, y_{j}\right) * \operatorname{MDC}\left(x_{i}, y_{j}\right)^{K}$

where $\operatorname{MDC}\left(x_{i}, y_{j}\right)^{K}$ is the $M D C$ value of the eight points around point $\left(x_{i}, y_{j}\right)$ for the $k$ th component, and is expressed as $\left\{M D C_{k}\left(x_{i-1}, y_{j-1}\right)\right.$, $\left.M D C_{k}\left(x_{i-1}, y_{j}\right),,,,, M D C_{k}\left(x_{i+1}, y_{j}\right), M D C_{k}\left(x_{i+1}, y_{j+1}\right)\right\}_{1 \times 8}^{T}$. The coefficient matrix $C\left(x_{i}, y_{j}\right)$ is expressed as

$$
C\left(x_{i}, y_{j}\right)=\left[\begin{array}{ccccc}
1 & x_{i-1} & \cdots & x_{i-1}^{2} y_{j-1} & x_{i-1} y_{j-1}^{2} \\
1 & x_{i-1} & \cdots & x_{i-1}^{2} y_{j} & x_{i-1} y_{j}^{2} \\
\vdots & \vdots & \ddots & \vdots & \vdots \\
1 & x_{i+1} & \cdots & x_{i+1}^{2} y_{j} & x_{i+1} y_{j}^{2} \\
1 & x_{i+1} & \cdots & x_{i+1}^{2} y_{j+1} & x_{i+1} y_{j+1}^{2}
\end{array}\right]^{-1}
$$

Because coefficient matrix $C\left(x_{i}, y_{j}\right)$ is independent of $\omega_{k}{ }^{-2}$ and $\operatorname{MDC}\left(x_{i}, y_{j}\right)^{K}$ is linear to $\omega_{k}{ }^{-2}$, the estimated $M D C_{k}\left(x_{i}, y_{j}\right)^{\prime}$ is also linear to $\omega_{k}{ }^{-2}$.

Based on the real value $\operatorname{MDC}_{k}\left(x_{i}, y_{j}\right)$ and the estimated value $M D C_{k}\left(x_{i}, y_{j}\right)^{\prime}$, the differential value $Z_{k}$ of point $\left(x_{i}, y_{j}\right)$ can be deduced

$Z_{k}\left(x_{i}, y_{j}\right)=\frac{\left(M D C_{k}\left(x_{i}, y_{j}\right)-M D C_{k}\left(x_{i}, y_{j}\right)^{\prime}\right)^{2}}{\sum_{i=1}^{E} \sum_{j=1}^{F}\left(M D C_{k}\left(x_{i}, y_{j}\right)-M D C_{k}\left(x_{i}, y_{j}\right)^{\prime}\right)^{2}}$

where $E$ and $F$ are the number of columns and rows of the measuring points.

Since both $\operatorname{MDC}_{k}\left(x_{i}, y_{j}\right)$ and $M D C_{k}\left(x_{i}, y_{j}\right)^{\prime}$ are linear to $\omega_{k}{ }^{-2}, Z_{k}$ is independent of natural frequency $\omega_{k}$ and only related to the $k$ th order mode shape.

As we know, influences of different damages on different order modes are quite divergent. The diversity of sensitiveness is especially obvious for specimens with multiple damages. Therefore, to identify various damages, the index $D I_{m}$ is defined,

$D I_{m}=\sum_{k=K_{1}}^{K_{2}} \alpha_{k}^{m} Z_{k}$ 


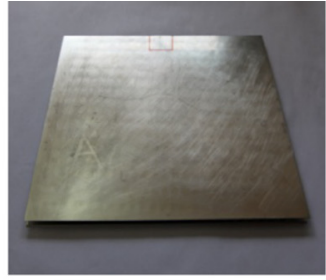

(a)

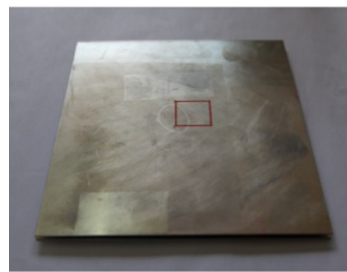

(e)

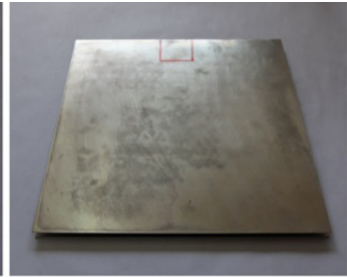

(b)

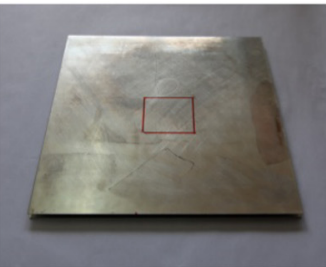

(f)

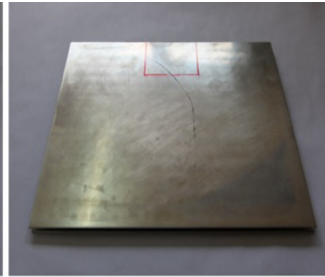

(c)

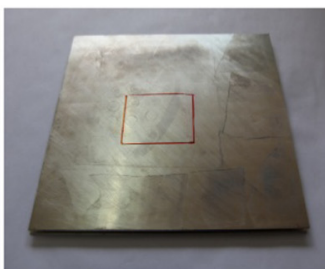

(g)

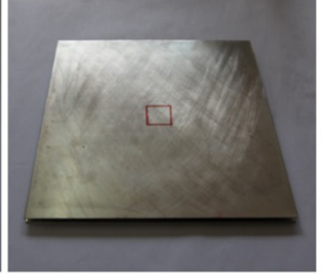

(d)

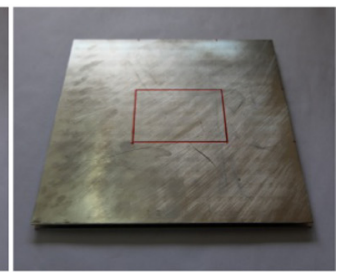

(h)

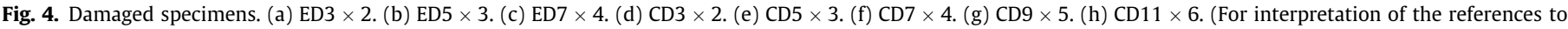
colour in this figure legend, the reader is referred to the web version of this article.)

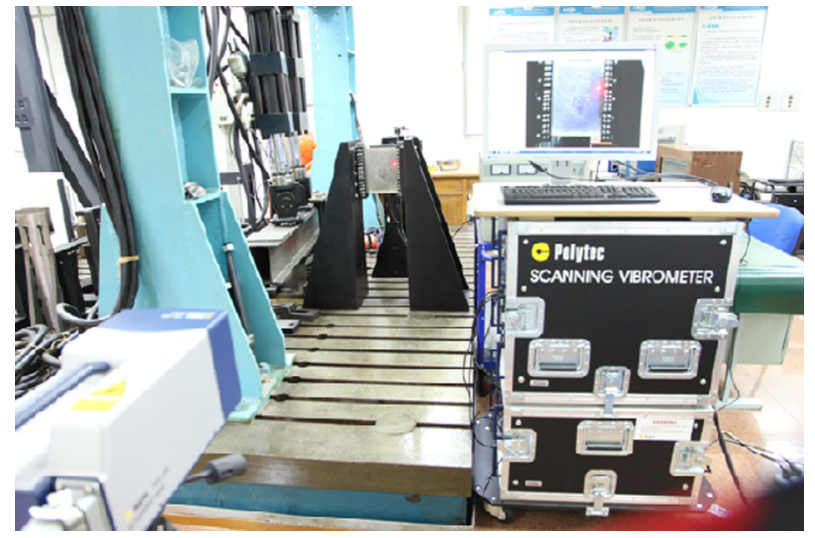

Fig. 5. Experimental setup.

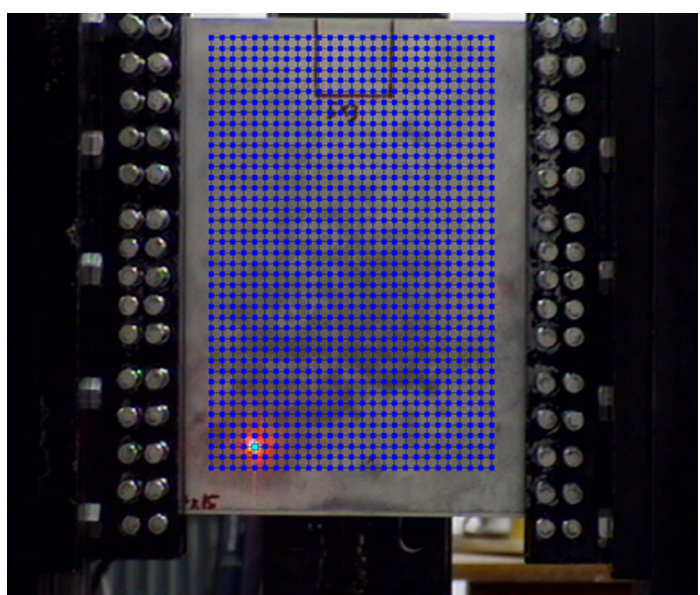

Fig. 6. Measuring points of ED $5 \times 3$ specimen.

where $K_{1}$ and $K_{2}$ are the beginning and the ending order modes in the practical case. $\alpha_{k}^{m}$ is defined as the weight coefficient of the $k$ th component and expressed as

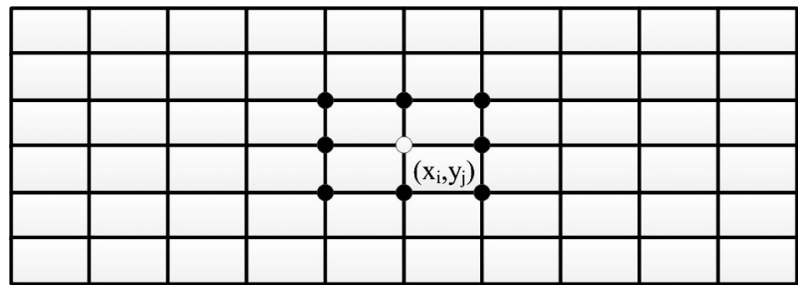

Fig. 7. Gapped grid points.

$\alpha_{k}^{m}=\omega_{k}^{m} / \sum_{k=K_{1}}^{K_{2}} \omega_{k}^{m}$

$\alpha_{k}^{m}$ could determine the weights of low order modes and high order modes in $D I_{m}$, and $m$ is an integer. When $m$ is less than 0 , the weight of low order modes in $D I_{m}$ is higher than high order modes. When $m$ is equal with 0 , the weight of low order modes in $D I_{m}$ is equal to high order modes. When $m$ is larger than 0 , the weight of low order modes in $D I_{m}$ is smaller than high order modes.

To suppress the fluctuations and singularities caused by nondamage factors (boundary conditions, noise, shaker, etc.), TEO is used to process the index $D I_{m}$, then the damage index $D I_{m}{ }^{*}$ can be obtained. The damage index $D I_{m}{ }^{*}$ for spatial sampling points can be expressed as Eq. (9), which is used to identify unbound nodes damages of metallic SPTCs.

$D I_{m}^{*}(n)=T\left(D I_{m}(n)\right)=D I_{m}^{2}(n)-D I_{m}(n+1) D I_{m}(n-1)$

\section{Experimental results and discussion}

In the work, damage index $D I_{0}^{*}$ are used to identify damages of the specimens. Since the eight specimens used in the study have only one damage location.

\subsection{Experimental results}

Based on the proposed method, $D I_{0}^{*}$ results of the eight damaged specimens are shown in Fig. 8. From Fig. 8(a)-(c), it is seen that all the unbound nodes at structural edge could be identified. It is also observed that the influence of the peak caused by the shaker on 


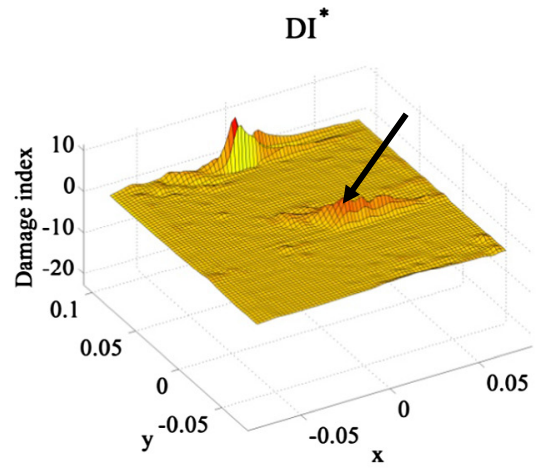

(a)

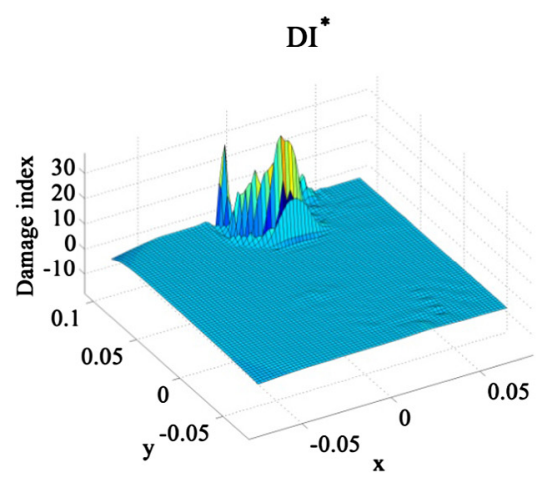

(c)

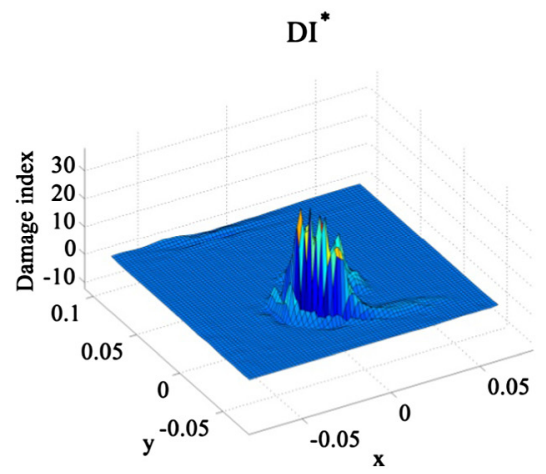

(e)

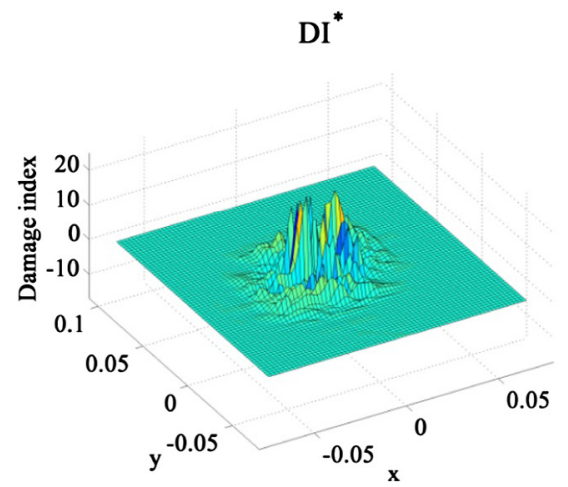

(g)

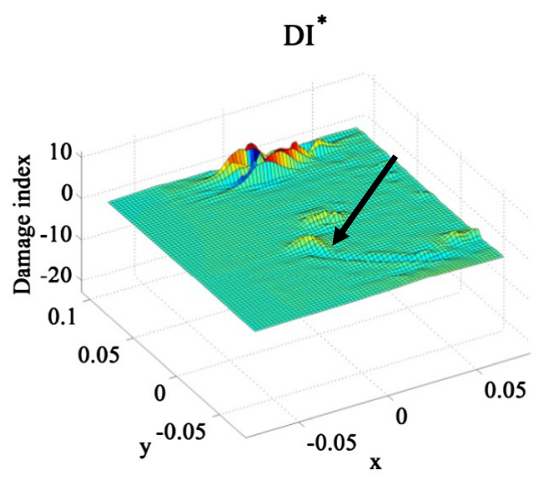

(b)

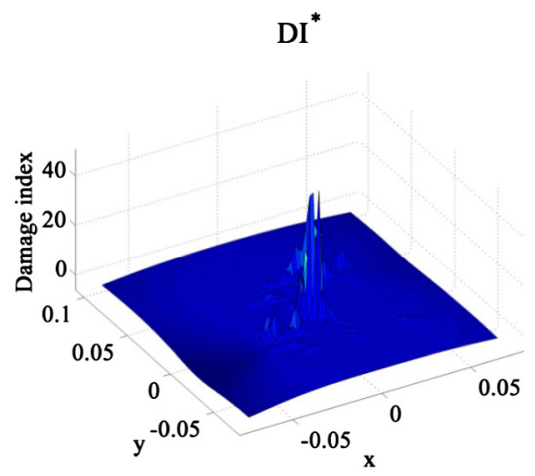

(d)

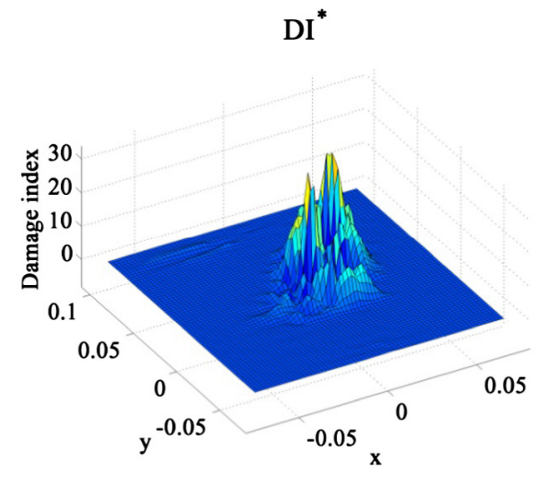

(f)

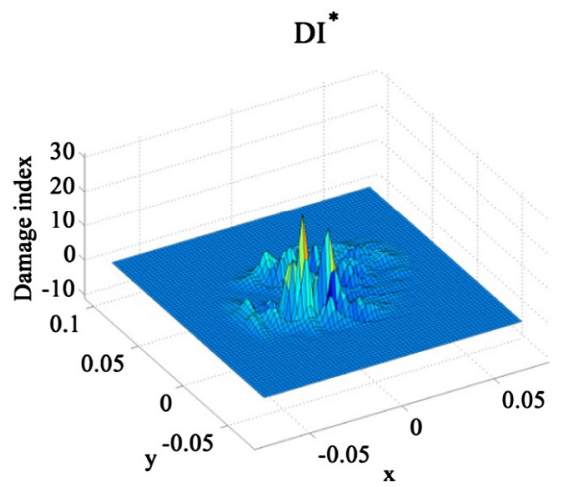

(h)

Fig. 8. $\mathrm{DI}_{0}^{*}$ results. (a) $\mathrm{ED} 3 \times 2$. (b) $\mathrm{ED} 5 \times 3$. (c) $\mathrm{ED} 7 \times 4$. (d) $\mathrm{CD} 3 \times 2$. (e) $\mathrm{CD} 5 \times 3$. (f) $\mathrm{CD} 7 \times 4$. (g) $\mathrm{CD} 9 \times 5$. (h) $\mathrm{CD} 11 \times 6$.

damage identification becomes invisible as the extent of the unbound nodes damage increases.
However, in Fig. 8(a) and (b), there is also a peak around the location of the shaker, as the black arrow pointed. This is because 
the shaker could cause the additional mass and local disturbance, and this disturbance can be observed when the size of actual damage is small. To differentiate the peak caused by the shaker with the peak caused by the real structural damages, performing an additional test in which the shaker is changed to another location may be a good solution. In the additional test, if there is no peak at the prior shaker location, it demonstrates that there is the only damage at the structural edge.

$D I_{0}^{*}$ results of specimens with the unbound nodes damage in the structural center are shown in Fig. 8(d)-(h). It is found that all different extent damages in the structural center could be identified by $D I_{0}^{*}$. Comparing $D I_{0}^{*}$ results of Fig. $8(\mathrm{a})$ and (d), (b) and (e), it is seen that it is more difficult to identify damage at edge than damage in structural center. This is because the location of the shaker is near the structural center, and it is easier to excite local vibration modes at damage zone in the center.

From Fig. 8(a)-(c) and (d)-(h), it is observed that the singularity zone of $D I_{0}^{*}$ results increases as the size of the actual unbound nodes zone increases. It is indicated that the proposed damage index $D I_{0}^{*}$ could reflect the size of the unbound nodes damage. Therefore an unbound nodes size identification method based on index $D_{m}{ }^{*}$ is developed and will be discussed in Section 5.4.

\subsection{Effect of sensor density}

The influence of sensor density on the unbound nodes detection accuracy is discussed in this section. Different sensor density, i.e., $31 \times 37,21 \times 25$ and $11 \times 15$ measuring points are conducted to detect the unbound nodes of the CD3 $\times 2$ specimen, and the sensor distributions in the case of different density are shown in Fig. 9(a)(c). It is seen that there are 25,9 and 1 measuring points dis- tributed in the unbound nodes zone respectively for the case of sensor density $31 \times 37,21 \times 25,11 \times 15$.

$D I_{0}^{*}$ results in the case of different sensor density are shown in Fig. 9(d)-(f). It is found that the unbound nodes damage could be localized accurately when the measurement point is more than $21 \times 25$. When the sensor density decreases to $11 \times 15$, the unbound nodes damage is influenced greatly by the fluctuation caused by boundary condition and noise (as the red circle shown in Fig. 9(f)) and could not be located accurately. Comparing $D I_{0}^{*}$ results in the case of different sensor density, it is found that the unbound nodes damage can be identified more accurately as the number of measuring points in the damage zone increases. According to the distribution of measuring points of $\mathrm{CD} 3 \times 2$ specimen, it is indicated that at least more than two measuring points should be arranged along one periodic length in order to obtain a good damage detection result.

\subsection{Effect of TEO}

To evaluate the effect of TEO on unbound nodes damage identification, $D I_{0}$ results of specimens with same size damages $(E D 3 \times 2$, ED5 $\times 3, C D 3 \times 2, C D 5 \times 3$ ) are supplied, as shown in Fig. 10 . According to $D I_{0}$ results in Fig. 10(a)-(b), it is observed that there are some fluctuations caused by the shaker (the red rectangle zone) and boundary conditions (the black arrow pointing), which influence the actual damage identification greatly. However, according to $D I_{0}^{*}$ results shown in Fig. 8(a), (b), (d) and (e), it is observed that the fluctuations and singularities caused by nondamage factors can be suppressed effectively and the real unbound nodes damage zone are emphasized by the damage index $D I_{0}^{*}$. It is indicated that TEO plays an important role in suppressing fluctua-

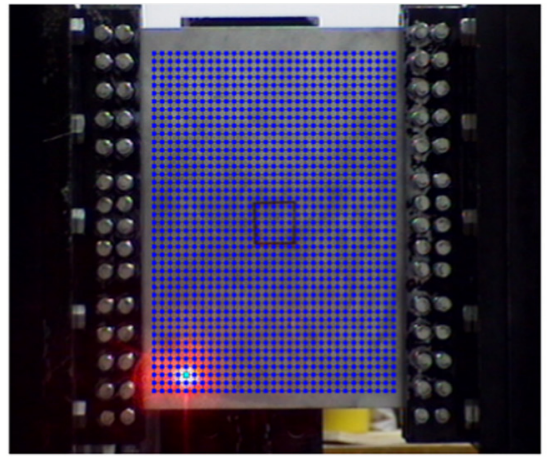

(a)

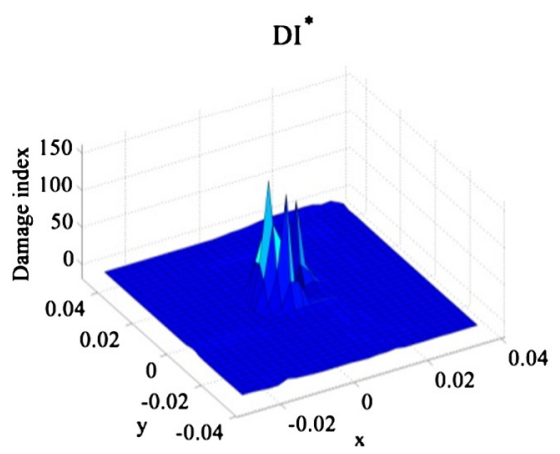

(d)

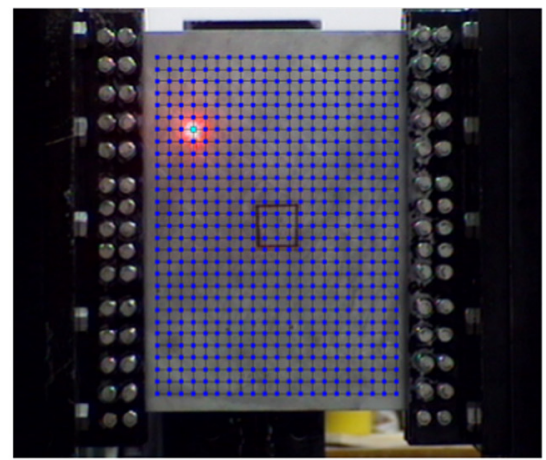

(b)

DI

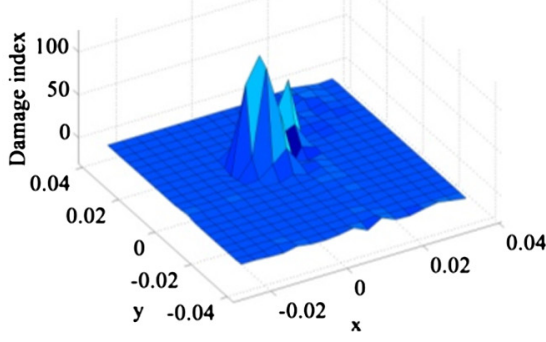

(e)

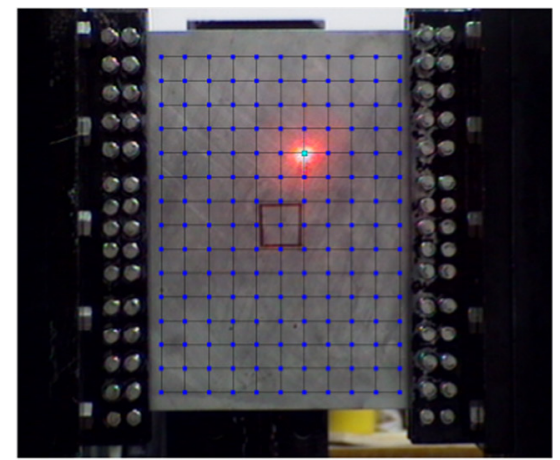

(c)

DI

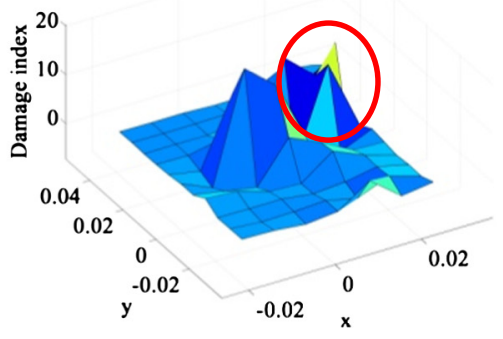

(f)

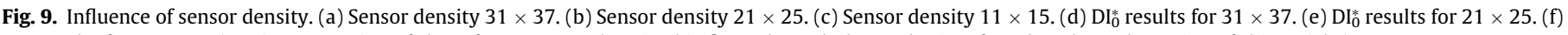
$\mathrm{DI}_{0}^{*}$ results for $11 \times 15$. (For interpretation of the references to colour in this figure legend, the reader is referred to the web version of this article.) 
DI

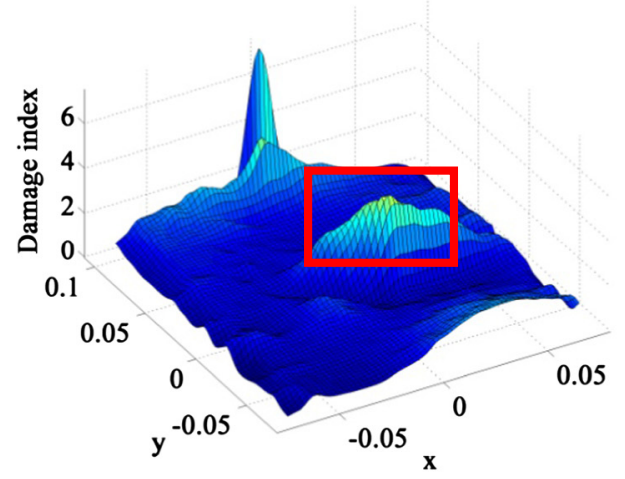

(a)

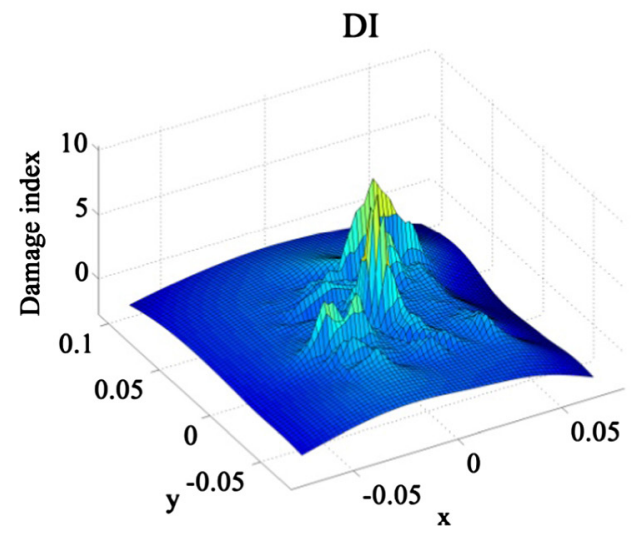

(c)

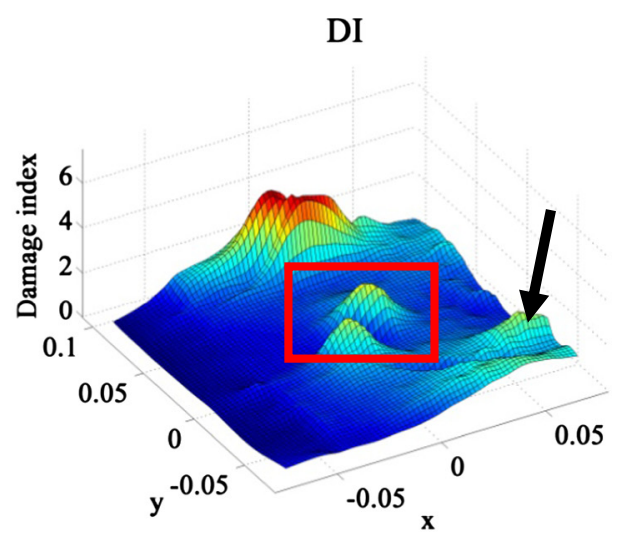

(b)

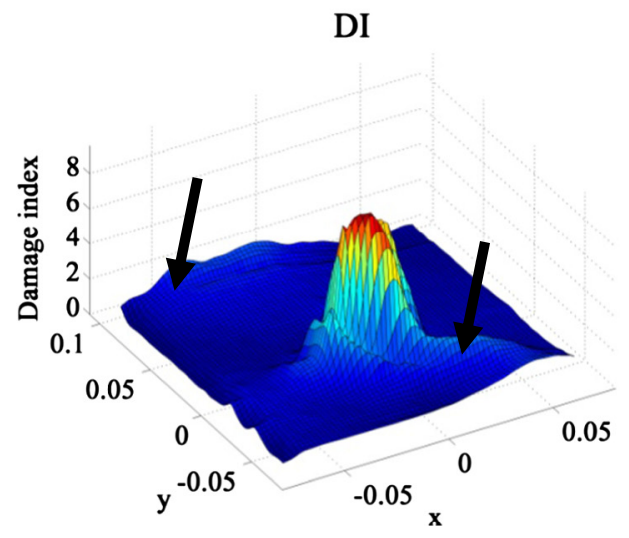

(d)

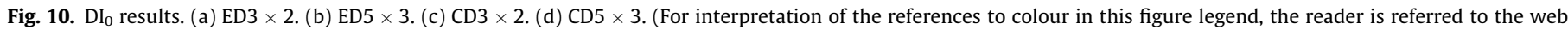
version of this article.)

tions and singularities of trend caused by non-damage factors in the experiments.

\subsection{Determining the size of unbound nodes zone}

For unbound nodes damage, there is no damage extent problem in the damage zone, i.e. the damage extent is same in the unbound nodes damage zone. Therefore, more attention should be paid to identify the shape or size of the unbound nodes zone, and determining the size of unbound nodes zone can be treated as a $0-1$ damage problem. Up to now, most proposed damage identification method focused on the detection of damage location, and little attention has been paid on the identification of damage shape or size. In the work, a 0-1 unbound nodes identification method is proposed to identify the boundary or size of the unbound nodes damage zone roughly. The method includes two steps:

Firstly, the damage index $D I_{m}{ }^{*}$ is integrated in the grid field of point $\left(x_{i}, y_{j}\right)$ and the obtained total $D I_{m}{ }^{*}$ value of point $\left(x_{i}, y_{j}\right)$ could be expressed as

$V_{D I_{m}^{*}}\left(x_{i}, y_{j}\right)=\int_{y_{1}}^{y_{2}} \int_{x_{1}}^{x_{2}} D I_{m}^{*}\left(x_{i}, y_{j}\right) d x d y$

where $x_{1}$ and $x_{2}$ are the beginning and ending $\mathrm{x}$ coordinate of the grid field of point $\left(x_{i}, y_{j}\right)$, and $y_{1}$ and $y_{2}$ are the beginning and ending $\mathrm{y}$ coordinate of the grid field of point $\left(x_{i}, y_{j}\right)$.

Secondly, the $0-1$ figure is obtained according to the $0-1$ evaluation criterion (Eqs. (11) and (12)). As we know, in the practical measurement, errors exist in the measuring results due to the noise, measurement error and so on. Normally, $5 \%$ is considered as the error limit. Therefore, if $V_{D I_{m}^{*}}\left(x_{i}, y_{j}\right)$ is not less than 5 percent of maximal $V_{D I_{m}^{*}}$ (as expressed in Eq. (11)), point $\left(x_{i}, y_{j}\right)$ is treated as in the unbound nodes zone, and the value of point $\left(x_{i}, y_{j}\right)$ is set 1 in the $0-1$ figure. If $V_{D I_{m}^{*}}\left(x_{i}, y_{j}\right)$ is less than 5 percent of maximal $V_{D I_{m}^{*}}$ (Eq. (12)), the point is treated as in an intact zone, and value of point $\left(x_{i}, y_{j}\right)$ is set 0 in the $0-1$ figure. After the calculation throughout the panel, the $0-1$ damage figures of specimens could be obtained and the size of unbound nodes zone could be identified.

$V_{D I_{m}^{*}}\left(x_{i}, y_{j}\right) \geqslant \max \left(V_{D I_{m}^{*}}\right) \times 5 \%$

$V_{D I_{m}^{*}}\left(x_{i}, y_{j}\right)<\max \left(V_{D I_{m}^{*}}\right) \times 5 \%$

Based on the proposed method, the integral results $V_{D I_{0}^{*}}$ of specimen CD5 $\times 3$, CD7 $\times 4$ and ED7 $\times 4$ are as shown in Fig. 11(a), (c) and (e). By processing $V_{D I_{0}^{*}}$ with the $0-1$ criterion, the $0-1$ unbound nodes figures of the three specimens are obtained, as shown in Fig. 11(b), (d) and (f). The blue field is non-damaged zone (NDZ) and the red field is the identified unbound nodes zone (IUNZ), and the yellow line is the boundary of prefabricated unbound nodes zone (PUNZ). It is observed from Fig. 11 that the location and size of the obtained unbound nodes zone could be in accordance with the actual damage zone basically. Because the distribution of measuring points is not cover all the unbound nodes zone for ED7 $\times 4$ (like Fig. 6 shown), the identified damage zone of ED7 $\times 4$ is smaller than CD7 $\times 4$. 


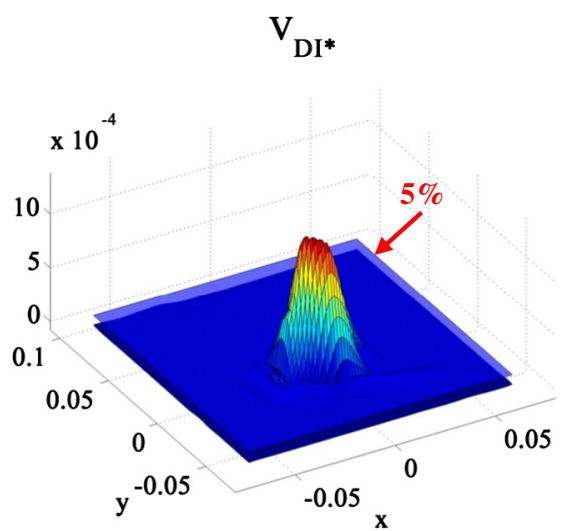

(a)

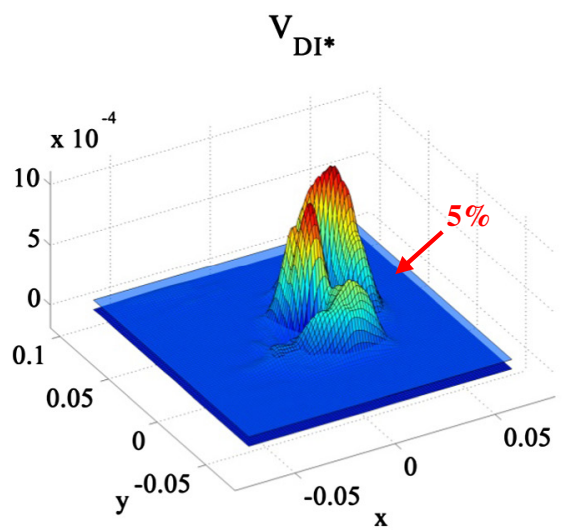

(c)

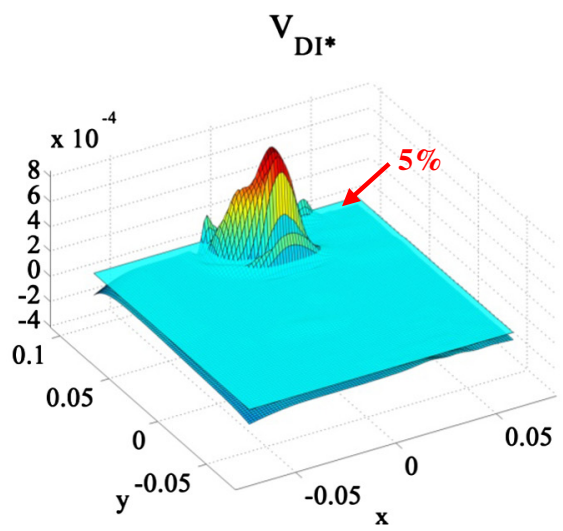

(e)

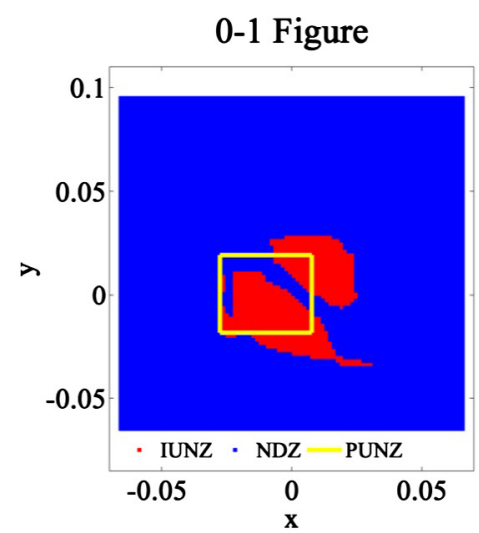

(b)

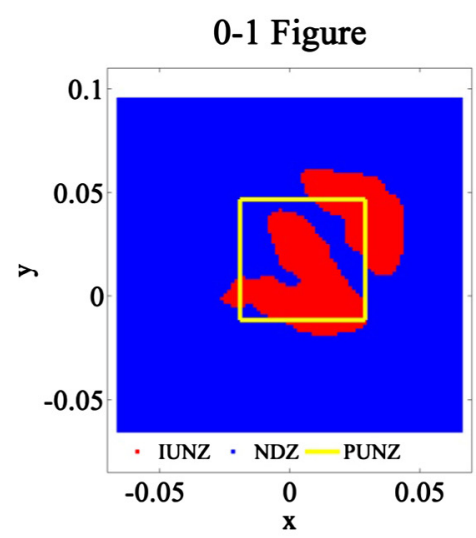

(d)

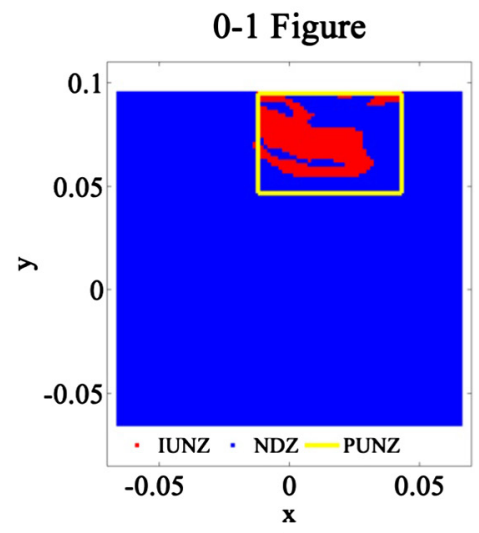

(f)

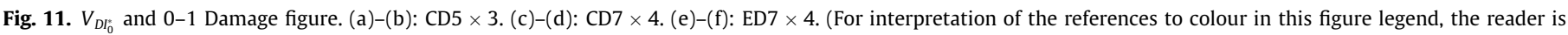
referred to the web version of this article.)

Generally, the $0-1$ figure obtained by the $0-1$ unbound nodes identification method could reflect the unbound nodes damage distinctly. However, the precision of the method need to be improved in the spatial domain.

\section{Conclusions}

In the paper, a baseline-free damage identification method is proposed to identify unbound nodes damage of metallic SPTCs. Experimental validations of various unbound nodes cases are conducted to demonstrate the effectiveness of the proposed method. Based on the results, some conclusions could be obtained:
The proposed damage index $D I_{m}{ }^{*}$ could detect unbound nodes damage effectively and the index could also reflect the size or extent of damages.

Increasing sensor density is beneficial to local unbound nodes damage identification and there is a critical sensor density for the damage identification.

By comparing $D I_{m}$ and $D I_{m}{ }^{*}$ results, it is found that TEO could effectively suppress fluctuations and singularities caused by nondamage factors, such as boundary conditions, noise and shaker and so on.

The proposed 0-1 unbound nodes identification method could be used to identify the size of unbound nodes damage zone, and 
the obtained 0-1 figure could reflect the unbound nodes damage distinctly. However, more efforts should be made to improve the spatial precision.

It is worth mentioning that structural geometric parameters of SPTCs, including thickness of face sheets, height of truss core, etc., would have effects on the damage identification result by the proposed method. For example, when the height of core part is constant, the influence of unbound nodes damage on dynamic properties of SPTCs would decrease as the thickness of face sheets increase. To investigate the influence of geometric parameters on damage identification, further works need to be carried out.

\section{Acknowledgements}

This research is supported by the National Natural Science Foundation of China under grant Nos. 11472276, 11332011 and 11502268, and National Defense Basic Scientific Research Program of China under grant No. JCKY2016130B009.

\section{References}

[1] Hg A. Analysis and design of structural sandwich panels. Oxford: Pergamon Press; 1969.

[2] Chiras S, Mumm DR, Evans AG, Wicks N, Hutchinson JW, Dharmasena K, et al. The structural performance of near-optimized truss core panels. Int J Solids Struct 2002;39:4093-115.

[3] Wadley HNG, Fleck NA, Evans AG. Fabrication and structural performance of periodic cellular metal sandwich structures. Compos Sci Technol 2003;63:2331-43.

[4] Zumpano G, Meo M. Damage detection in an aircraft foam sandwich panel using nonlinear elastic wave spectroscopy. Comput Struct 2008;86:483-90.

[5] Panopoulou A, Loutas T, Roulias D, Fransen S, Kostopoulos V. Dynamic fiber Bragg gratings based health monitoring system of composite aerospace structures. Acta Astronaut 2011;69:445-57.

[6] Evans AG, Hutchinson JW, Fleck NA, Ashby MF, Wadley HNG. The topological design of multifunctional cellular metals. Prog Mater Sci 2001;46:309-27.

[7] Wang J, Lu TJ, Woodhouse J, Langley RS, Evans J. Sound transmission through lightweight double-leaf partitions: theoretical modeling. J Sound Vib 2005;286 (4-5):817-47.

[8] Evans AG, Hutchinson JW, Ashby MF. Multifunctionality of cellular metal systems. Prog Mater Sci 1998;43(3):171-221.

[9] Liu JY, Xiang LL, Kan T. The effect of temperature on the bending properties and failure mechanism of composite truss core sandwich structures. Compos Part A 2015;79:146-54.

[10] Liu JY, Qiao WF, Liu JX, Xie D, Zhou ZG, Wu LZ, et al. High temperature indentation behaviors of carbon fiber composite pyramidal truss structures. Comput Struct 2015;131:266-72.

[11] Yuan W, Wang X, Song HW, Huang CG. A theoretical analysis on the thermal buckling behavior of fully-clamped sandwich panels with truss cores. J Therm Stresses 2014;37:1433-48.
[12] Yuan W, Song HW, Wang X, Huang CG. Experimental investigation on thermal buckling behavior of fully-clamped truss-core sandwich panels. AIAA J 2015;53(4):948-57.

[13] Lu LL, Song HW, Huang CG. Effects of random damages on dynamic behavior of metallic sandwich panel with truss core. Comp Part B 2016.

[14] Wallach JC, Gibson LJ. Defect sensitivity of a 3D truss material. Scripta Mater 2001;45:639-44.

[15] Yuan W, Song HW, Lu LL, Huang CG. Effect of local damages on the buckling behavior of pyramidal truss core sandwich panels. Compos Struct 2016;149:271-8.

[16] Russell B, Hilary BS. Imperfection sensitivity of pyramidal core sandwich structures. Int J Solids Struct 2007;44:4690-706.

[17] Hu JS, Hwu C. Free vibration of delaminated composite sandwich beams. AIAA J 1995;33(10):1911-8.

[18] Kim HY, Hwang W. Effect of debonding on natural frequencies and frequency response functions of honeycomb sandwich beams. Compos Struct 2002;55:51-62.

[19] Sokolinsky VS, Bremen HF, Lesko JJ, Nutt SR. Higher-order free vibrations of sandwich beams with a locally damaged core. Int J Solids Struct 2004;41:6529-47.

[20] Burlayenko VN, Sadowski T. Influence of skin/core debonding on free vibration behavior of foam and honeycomb cored sandwich plates. Int J Non-Linear Mech 2010;45(10):959-68.

[21] Buket OB, Srinivasa T. An experimental investigation of free vibration response of curved sandwich beam with face/core debond. J Reinf Plast Comp 2010 (00):1-11.

[22] Lou J, Wu LZ, Ma L, Xiong J, Wang B. Effects of local damage on vibration characteristics of composite pyramidal truss core sandwich structure. Compos Part B 2014;62:73-87.

[23] Lestari W, Qiao P. Damage detection of fiber-reinforced polymer boneycomb sandwich beams. Compos Struct 2005;67:365-73.

[24] Hu HW, Wang BT, Lee CH, Su JS. Damage detection of surface cracks in composite laminates using modal analysis and strain energy method. Compos Struct 2006;74:399-405.

[25] Kumar M, Shenoi RA, Cox SJ. Experimental validation of modal strain energies based damage identification method for a composite sandwich beam. Compos Sci Technol 2009;69:1635-43.

[26] Zhu KG, Chen MJ, Lu QH, Wang B, Fang DN. Debonding detection of honeycomb sandwich structures using frequency response functions. J Sound Vib 2014;333:5299-311.

[27] Andrzej K. Vibration-based spatial damage identification in honeycomb-core sandwich composite structures using wavelet analysis. Compos Struct 2014;118:385-91.

[28] Tian SX, Chen ZM, Chen LL, Zhang ZL. Numerical analyses on influence of damage configuration on vibration parameters for lattice sandwich plate. Int J Appl Electromagnet Mech 2010;33:1565-72.

[29] Li B, Li Z, Zhou J, Ye L, Li E. Damage localization in composite lattice truss core sandwich structures based on vibration characteristics. Compos Struct 2015;126:34-51.

[30] Lu LL, Song HW, Yuan W, Huang CG. Baseline-free damage identification of metallic sandwich panels with truss core based on vibration characteristics. Struct Health Monit 2016:1-15.

[31] Ratcliffe CP. Damage detection using a modified laplacian operator on mode shape data. J Sound Vib 1997;204:505-17.

[32] Yoon MK, Heider D, Gillespie JW, Ratcliffe CP, Crane RM. Local damage detection using the two-dimensional gapped smoothing method. J Sound Vib 2005;279:119-39. 